\title{
¿Cuánto saben sobre heridas crónicas los estudiantes de Grado de Enfermería de la Comunidad de Madrid?
}

\author{
Cristina Sánchez Lluch ${ }^{1}$, Marta Martín Álvarez ${ }^{1}$, Luis Ernesto Garrido Romero ${ }^{2}$ \\ ${ }^{1}$ Estudiante de 3o de Grado de Enfermería de la Universidad Complutense de Madrid (UCM). España \\ ${ }^{2}$ Estudiante de 10 de Grado de Enfermería de la Universidad Europea de Madrid. España
}

\author{
Correspondencia \\ Cristina Sánchez Lluch \\ Correo electrónico: cris_lluch@hotmail.com \\ (Recibido: 09/05/2018. Aceptado: 30/05/2018)
}

\section{RESUMEN}

Una herida representa la pérdida de la continuidad de la piel. Las heridas crónicas son una importante causa de morbilidad y mortalidad en todo el mundo, y pueden producirse por múltiples causas. La edad, fármacos consumidos por el paciente, tabaquismo, la malnutrición, obesidad, sedentarismo, situación sociofamiliar, patologías asociadas y tratamiento inadecuado de la herida son factores que van a repercutir negativamente en la cicatrización adecuada. El proceso de la cicatrización es el resultado de una interacción compleja entre los factores del paciente y de la herida, el tratamiento empleado y los conocimientos y habilidades de los profesionales sanitarios. Cuando se supera el periodo de 6 semanas las heridas comienzan a considerarse crónicas. A pesar de los esfuerzos por implementar la evidencia científica, en la práctica de enfermería aún se observa una separación entre lo académico y lo clínico; esta separación viene dada sobre todo por la actitud del profesional, la falta de recursos y apoyo que impiden que la investigación se utilice para tomar decisiones en la práctica clínica. El objetivo de este estudio era evaluar el conocimiento de los alumnos de cuarto de enfermería sobre heridas. Se realizó un estudio descriptivo y transversal, durante el mes de abril de 2018, entre estudiantes de 4o de Enfermería de la Comunidad de Madrid. Se elaboró una encuesta ad hoc que fue difundida por medios telemáticos entre estudiantes de universidades públicas y privadas. Para el análisis de resultados se llevó a cabo un análisis estadístico descriptivo con cálculo de frecuencias absolutas y relativas para las variables cualitativas y media con desviación estándar para las variables cuantitativas. El estudio estadístico se realizó con el programa Access. Los estudiantes entrevistados tienen un nivel de conocimientos insuficiente, pero sería necesario llevar a cabo estudios adicionales que confirmen estos resultados. Nuestro compromiso es llevar a cabo un estudio más amplio, cuando nuestras competencias investigadoras lo permitan, que además ayude a identificar posibles mejoras en la formación que reciben los alumnos en este campo.

\section{PALABRAS CLAVE}

Úlcera cutánea; Estudiantes de enfermería; Capacitación profesional

\section{SUMMARY}

A wound represents the loss of skin continuity. Chronic wounds are an important cause of morbidity and mortality throughout the world, and can occur from multiple causes. Age, medicines consumed by the patient, smoking, malnutrition, obesity, sedentary lifestyle, socio-familial situation, associated pathologies and inadequate treatment of the wound are factors that will have a negative impact on adequate healing.

The healing process is the result of a complex interaction between the patient's and the wound's factors, the treatment used and knowledge and skills of health professionals.

When the period of 6 weeks is exceeded, wounds begin to be considered chronic. Despite the efforts to implement scientific evidence, in nursing practice, there is still a separation between academic and clinical; This separation is mainly due to the attitude of the professional, the lack of resources and support that prevent the research from being used to make decisions in clinical practice.

The objective of this study was to assess the knowledge of nursery students about injuries. A descriptive and crosssectional study was conducted, during the month of April 2018, among students of 4th Nursing in the Community of Madrid. An ad hoc survey was developed and disseminated by telematic means among students of public and private universities.

For the analysis of results, a descriptive statistical analysis was carried out with calculation of absolute and relative frequencies for the qualitative variables and mean with standard deviation for the quantitative variables.

The statistical study was carried out with the Access program. The students interviewed have an insufficient level of knowledge, but it would be necessary to carry out additional studies that confirm these results. 
[Cristina Sánchez Lluch, et al]

¿Cuánto saben sobre heridas crónicas los estudiantes de Grado de Enfermería de la Comunidad de Madrid?

Our commitment is to carry out a broader study, when our research competences allow it, which also helps to identify possible improvements in the training that students receive in this field.

\section{KEYWORDS}

Cutaneous ulcer; Nursing students; Professional training

\section{Introducción}

Las heridas crónicas son una importante causa de morbilidad y mortalidad en todo el mundo, pueden producirse por múltiples causas, entre ellas la diabetes mellitus, la neuropatía periférica, la inmovilidad, la presión, la arteriosclerosis, las infecciones y/o la insuficiencia venosa (1).

El cuidado de las heridas crónicas supone un reto para los profesionales de enfermería. Las diferentes etiologías responsables de las heridas crónicas, el elevado número de productos existentes en el mercado, así como la práctica no contrastada con evidencias científicas (2) hacen que nos encontremos con una alta variabilidad de los cuidados enfermeros. Los métodos de diagnóstico y evaluación de las heridas y su cicatrización no han tenido el mismo desarrollo en el tiempo. La cicatrización no ha sido un proceso que se valore de manera adecuada en las heridas, en especial en las heridas crónicas $(\mathrm{HC})$. Generalmente, estas lesiones han carecido de interés para los profesionales sanitarios, que siempre las han considerado como situaciones normales e irremediables en determinadas patologías (3). El estudio de Esperon (2) establece que la variabilidad en el tratamiento de las heridas parece relacionarse, principalmente, con un déficit de formación (35,6\%) y con las actitudes de los profesionales (22,3\%) que las atienden más que con las necesidades de la población atendida (4,5\%).

Una herida representa la pérdida de la continuidad de la piel. Pueden clasificarse en agudas o crónicas en función del tiempo de cicatrización (5).Las heridas crónicas son aquellas lesiones que presentan una escasa o nula tendencia a la cicatrización. Cuando se supera el periodo de 6 semanas comienzan a considerarse crónicas (6).

El proceso de la cicatrización es el resultado de una interacción compleja entre los factores del paciente y de la herida, el tratamiento empleado y los conocimientos y habilidades de los profesionales sanitarios (4). La edad, fármacos consumidos por el paciente, tabaquismo, la malnutrición, obesidad, sedentarismo, situación sociofamiliar, patologías asociadas y tratamiento inadecuado de la herida son factores que van a repercutir negativamente en la cicatrización adecuada (1).

El daño en la piel puede tener un impacto perjudicial en el bienestar general del paciente (1). Los pacientes que presentan este tipo de heridas acuden a diario al centro de salud o, en el caso de estar inmovilizados, es el personal de enfermería el que se desplaza a sus domicilios. (1)

Para un correcto abordaje sanitario de las heridas crónicas y mejora de la cicatrización de estas, en una valoración inicial se debe valorar y registrar la antigüedad de la lesión, localización, estadio en la que se encuentra, dimensiones, tunelización, estado de la piel perilesional, cantidad y tipo de exudado, signos de infección, presencia de dolor y olor. Una vez identificado esto se debe consensuar el tipo de cura a realizar y la periodicidad. (5)(3).La cura incluye el desbridamiento, ya sea quirúrgico, enzimático, autolítico, osmótico y mecánico y la elección del apósito en función del exudado (7). Un estudio realizado en Estados Unidos (8) en 400 pacientes con UPP, úlceras venosas y de pie diabético entre 1999-2004/2005 sugiere que muchos elementos de la valoración de las heridas crónicas son conocidos y puestos en práctica (tipo de herida, localización, cantidad de exudado, presencia de necrosis). Sin embargo, otros elementos de la valoración no están sistemáticamente documentados: tejido del lecho, bordes, profundidad, color de la piel perilesional o presencia de edema; y en cuanto a la coherencia del tratamiento seleccionado con las prácticas recomendadas se observa que el uso de agua del grifo o solución salina para la limpieza de las heridas presenta niveles bajos de seguimiento, y en cuanto a la elección de apósitos algunos profesionales nunca utilizan apósitos de cura en ambiente húmedo (hidrocoloides, hidrofibras, hidrogel, alginato, espumas, colágeno, etc.) o realizan una selección inadecuada de estos destacando la aplicación de apósitos para el manejo del exudado en heridas no exudativas.

A pesar de los esfuerzos por implementar la evidencia científica, en la práctica de enfermería aún se observa una separación entre lo académico y lo clínico; esta separación viene dada sobre todo por la actitud del profesional, la falta de recursos y apoyo que impiden que la investigación se utilice para tomar decisiones en la práctica clínica (5). Disminuir la variabilidad en los cuidados enfermeros que proporcionamos es una responsabilidad no solo de los profesionales, que tienen la obligación y el derecho de formarse, sino también de la administración sanitaria, que debe facilitar y potenciar las actividades formativas para garantizar la competencia de los profesionales y una atención sanitaria de calidad (9).

Otro aspecto que debe contemplarse al evaluar el déficit de formación es la realizada a los alumnos de enfermería. Moore y Clarke (2009) (10), en una encuesta realizada en 28 países, hallaron que esta formación varía considerablemente tanto en el tiempo dedicado al abordaje de las heridas crónicas (desde 2 horas a 1 día) como en el programa, manifestando el $80 \%$ de los encuestados su discrepancia con el tiempo dedicado durante la formación enfermera al manejo de heridas y 
considerando en general que los estudiantes de enfermería no están adecuadamente preparados para la práctica asistencial de las mismas.

El objetivo de este estudio es realizar una valoración del nivel de conocimiento que tiene los alumnos de cuarto de enfermería de la Comunidad Autónoma de Madrid (CAM) sobre este tema.

\section{Método}

Se ha realizado un estudio descriptivo y transversal durante el mes de abril de 2018, entre estudiantes de 40 de Enfermería de las universidades siguientes: Universidad Complutense de Madrid, Universidad Europea de Madrid, Universidad Autónoma de Madrid, Univer- sidad Francisco de Vitoria, Universidad San Juan de Dios, EU de Enfermería de la Cruz Roja, EU de Enfermería Fundación Jiménez Díaz y Universidad Rey Juan Carlos, todas ellas de la Comunidad de Madrid. El contacto con los alumnos se realizó a través de varias asociaciones de estudiantes, las cuales revisaron el cuestionario previamente. Los estudiantes pertenecían tanto a universidades públicas como privadas. Se elaboró una encuesta ad hoc, formada por 10 preguntas (Fig. 1), con cuatro posibles respuestas y solo una de ellas verdadera, sobre conocimientos en heridas crónicas: etiología, tratamiento y elección de este, diagnóstico, clasificaciones y factores relevantes de salud. Para confeccionar dicha encuesta se consultaron diferentes

\section{¿Cuánto sabes de Heridas Crónicas?}

Solo para estudiantes de $4^{\circ}$ curso Grado de Enfermería

\section{¿Eres alumno de 4ำ curso Grado de Enfermería?*}

\section{$\mathrm{Si}$}

1. Cuando una herida requiere tiempos prolongados para su cicatrización. ¿a partir de cuantas semanas se considera crónica, según la mayoría de la bibliografía?
$O$ a) 3 semanas
b) 4 semanas
c) 6 semanas
c) 5 semanas

2. Según clasificaciones, las úlceras arteriales:

$O$ a) Es el resultado de una isquemia severa prolongada en el tiempo, este tipo de heridas tienen mal pronóstico y su incidencia es más alta en varones mayores de 60 años

b) Se producen por incompetencia anatómica y funcional de las válvulas, dificultando el retorno de la sangre

$\bigcirc$ c) Se producen por una extravasación de líquido al tejido intersticial

$\bigcirc$ d) Ninguna es correcta

3. ¿Con cuál de los siguientes tipos de problemas, se relacionan las heridas crónicas?

$O$ a) Envejecimiento y problemas que conllevan asociados largos tiempos de inmovilidad

b) Fracturas de huesos cortos

c) Hiperemia

$O$ d) A y B son correctas

4. ¿Cuántos tipos de desbridamiento podemos encontrar?
a) Quirúrgico, enzimático y autolítico
b) Autolítico y quirúrgico
c) Osmótico, mecánico y larval
$\mathrm{O}$ d) A y $\mathrm{C}$ son correctas

5. Encontraremos los pulsos distales ausentes en

a) Úlcera varicosa y úlcera postrombótica.

b) Úlcera isquémica y úlcera neuropática.

$\bigcirc$ c) Solamente en la úlcera isquémica.

d) Solamente en la úlcera neuropática.

6. Utilizaremos la colagenasa cuando:

$O$ a) Existe placa necrótica

b) Junto con un hidrocoloide o espuma

$O$ c) Cuando existen esfacelos

$O$ d) En una úlcera infectada

7. ¿Cuándo debemos curar una úlcera infectada)

$O$ a) Cada 24 horas

b) Cada 2-3 días

c) cada 72 horas

d) Cada 24horas o se sature el apósito.

8. Para un exudado muy alto utilizaremos:

$\mathrm{O}$ a) Hidrogeles e hidrocoloides

b) Espumas

c) Hidrocoloides y espumas

$\bigcirc$ d) Alginatos

9. Según la clasificación de Fontaine aparecerá dolor en reposos en el estadía:
a) I
b) Ila y $\| b$
$\mathrm{O}$ c) III
O d) IV

10. Para las úlceras necróticas del pie diabético elegiremos:
$\mathrm{O}$ a) Desbridamiento autolítico con hidrogel
b) Hidrogeles y alginatos
$\mathrm{Oc}$ Solo hidrogeles
d) Ninguna delas respuestas es correcta

Fig. 1: Encuesta ad hoc de elaboración propia. 
Guías de Práctica Clínica, así como las recomendaciones sobre úlceras cutáneas crónicas de la CAM, además de otros artículos y protocolos basados en la evidencia científica. Esta encuesta fue difundida por medios telemáticos, a través de formularios Google, de manera online. El cuestionario estuvo abierto para acceder y responder durante 15 días, siendo solo posible responder una vez por persona. Para el análisis de resultados se llevó a cabo un análisis estadístico descriptivo con cálculo de frecuencias absolutas y relativas para las variables cualitativas y media con desviación estándar para las variables cuantitativas. El estudio estadístico se realizó con el programa Access.

\section{Resultados}

El cuestionario fue cumplimentado por 157 estudiantes, de los cuales el $41^{\prime} 42 \%(n=65)$ eran hombres.

Por universidades, el $46^{\prime} 49 \%(n=73)$ pertenecían a la universidad de Complutense de Madrid, el 13'38\% $(n=20)$ de la universidad Europea de Madrid, el 4,45\% $(n=7)$ de la universidad Autónoma de Madrid, el 11,46\% $(n=18)$ de la universidad Francisco de Vitoria, el 10'83\% $(n=17)$ de la universidad San Juan de Dios, el 9'55\% $(n=6)$ de la universidad EU de enfermería de la Cruz Roja, el 3'18\% ( $n=5)$ de la EU de Enfermería Fundación Jiménez Díaz y 10 el 6’37\% ( $n=10)$ de la universidad Rey Juan Carlos. El $70 \%(n=110)$ de los alumnos pertenecían a universidades privadas.

Se obtuvieron los siguientes resultados:

Un 59,6\% ( $n=38)$ de los estudiantes sabe que el criterio necesario para considerar una herida como crónica es que el tiempo de cicatrización supere las 6 semanas.

Un $87,7 \%(n=56)$ de los participantes sabe que las úlceras crónicas arteriales son el resultado de una isquemia severa y prolongada en el tiempo.

Un 75,4\% ( $n=48)$ sabe que los problemas de salud que más se asocian a las heridas crónicas son el envejecimiento y los problemas relacionados con la inmovilidad.

Un $42,1 \%(n=27)$ conoce todos los tipos de desbridamiento: quirúrgico, enzimático, autolítico, osmótico, mecánico y larval.

Un 45,6\% (n=29) sabe que es en la úlcera isquémica en la que encontramos ausentes los pulsos distales, mientras que otro $45,6 \%(n=29)$ dijo que la correcta era ulcera isquémica junto con la ulcera neuropática.

Un $31,6 \%(n=20)$ utilizaría colagenasa para desbridar placas necróticas.

Un $66,7 \%$ ( $n=42$ ) sabe que la periodicidad para curar una úlcera infectada es a diario o cuando el apósito se sature. Un $7 \%$ respondió que cada 72 horas y el resto otras respuestas.

Un $45,6 \%(n=29)$ sabe que ante exudados elevados se deben utilizar alginatos.

Un 31,6\% ( $n=20)$ identificó el dolor en reposo con el estadio III de la clasificación de Fontaine.

Un 47,4\% respondió que en la pregunta sobre desbridamiento de úlceras de pie diabético ninguna de las respuestas ofrecidas era correcta.

\section{Discusión}

Las heridas son un problema de salud al que las enfermeras nos enfrentamos de forma cada vez más frecuente en nuestra práctica clínica, afectan a una amplia población de todas las edades y requieren diferentes intervenciones para asegurar un cuidado óptimo de los pacientes que las presentan (11).

Con este estudio queríamos conocer el grado de conocimientos que tienen los alumnos de 4 o curso de grado de enfermería de las distintas universidades de la CAM sobre heridas crónicas.

A partir de los resultados obtenidos, cabe pensar que los conocimientos acerca de heridas crónica son deficientes entre estos alumnos $y$, por tanto, entre los futuros enfermeros, datos que se corresponden con los hallazgos de Moore y Clarke en 2011 (10).

La principal fortaleza de este estudio es que incluye a estudiantes de universidades tanto públicas como privadas

El estudio presenta ciertas limitaciones derivadas tanto del tamaño muestral como de la técnica de muestreo, por lo que los resultados no son concluyentes y resultaría necesario seguir investigando para ver si se confirman estos indicios. De cara a futuros estudios, además de seleccionar una muestra más amplia y aleatorizada, también sería muy útil realizar una encuesta que valorase la percepción que tienen los alumnos sobre los contenidos teóricos y prácticos que reciben durante su formación de grado.

Los hallazgos del estudio de Kim 2010 (12), destacaban la necesidad de implementar un enfoque integral para aumentar los conocimientos de prevención y manejo de lesiones por presión de los estudiantes australianos de enfermería, así como garantizar que estos estudiantes tengan experiencias adecuadas en unidades clínicas, con un alto enfoque en la prevención de lesiones. Nuestro compromiso es llevar a cabo un estudio más amplio, cuando nuestras competencias investigadoras lo permitan, que además ayude a identificar posibles mejoras en la formación que reciben los alumnos en este campo.

Mientras tanto, nos gustaría invitar a la reflexión sobre la formación que se imparte actualmente en este ámbito en la universidad, ya que como hemos señalado, el grado de conocimientos obtenidos por los alumnos parece inferior a lo esperado. Debido al envejecimiento de la población y al progresivo incremento de la prevalencia de factores de riesgo relacionados, la prevalencia de heridas crónicas irá ascendiendo exponencialmente en los próximos años, de modo que si es cierto que los futuros profesionales no están preparados para abordar adecuadamente el cuidado de estos pacientes, probablemente aumentarán también las complicaciones derivadas de estas lesiones y los tiempos de cicatrización se prolongarán, con la consiguiente repercusión sobre el gasto sanitario y la calidad de vida de los pacientes. 


\section{Conclusiones}

Las heridas son un problema de salud al que las enfermeras nos enfrentamos a diario en nuestra práctica clínica. Debido al paulatino envejecimiento de la población y a la amplia gama de productos disponibles, es necesario que los futuros profesionales sepan abordar esta problemática adecuadamente. Por todo ello, las instituciones docentes deben promocionar y facilitar la formación de los estudiantes en este campo.

Con este estudio pretendíamos realizar una valoración del nivel de conocimientos que tienen los estudiantes de Grado de Enfermería de la Comunidad de Madrid sobre heridas crónicas. Los estudiantes entrevistados tienen un nivel de conocimientos insuficiente, lo que se corresponde con los datos encontrados en la literatura científica, pero para poder generalizar estos resultados es necesario realizar estudios adicionales y de mayor calidad que los confirmen.

\section{Bibliografía}

1. González-Consuegra RV, Verdú Soriano J. Quality of life related with chronic wounds. Gerokomos. 2010; 21 (3): 131-139.

2. Esperón Güimil JA, Loureiro Rodríguez MT, Antón Fuentes MV, Rosendo Fernández JM, Pérez García I, Soldevilla-Agreda JJ. Variabilidad en el abordaje de las heridas crónicas: ¿qué opinan las enfermeras? Gerokomos [Internet]. 2014 Dic [citado 2018 Mayo 08]; 25(4): 171-177. Disponible en:

http://scielo.isciii.es/scielo.php?script=sci_arttext\& pid= S1134-928X2014000400007\&lng=es

3. Restrepo-Medrano JC, Verdú Soriano J. Desarrollo de un índice de medida de la evolución hacia la cicatrización de las heridas crónicas. Gerokomos [Internet]. 2011 [citado 2018 May 06]; 22(4):176-83. Available from: http://scielo.isciii.es/scielo.php? script=sci_arttext\&pid=S1134928X2011000400005 \&lng=en\&nrm =iso\&tlng=en

4. European Wound Management Association (EWMA). Documento de posicionamiento: Heridas de difícil cicatrización: un enfoque integral. Londres: MEP Ltd, 2008.

5. Servicio Madrileño de Salud. Recomendaciones para el tratamiento local de las úlceras cutáneas crónicas de la Comunidad de Madrid. Madrid: Comunidad de Madrid. Consejería de Sanidad; 2010.

6. Giménez-Medina RA, Díaz-Valenzuela A. Variabilidad de la práctica clínica enfermera en el abordaje de heridas crónicas en la Agencia Sanitaria Alto Guadalquivir. 2018;

7. Lorenzo M, Hernández R, Soria M. Heridas crónicas atendidas en un servicio de urgencias. Enfermería Global. 2014;(35):23-31.

8. Jones KR, Fennie K, Lenihan A. Evidence-Based Management of Chronic Wounds. Adv Skin Wound Care. 2007; 20:591-600. Disponible en: www.woundcarejournal.com

9. García Díaz J, Muñoz Conde A, Cabello Jaime R. Disminución de la Variabilidad Clínica a través de la Formación a Referentes en Úlceras por Presión. Bibl Lascasas. 2013; 9(1):aprox. 4[pantallas].

10. Moore Z. Clarke E. A survey of the provision of education in wound Management to undergraduate nursing students. EWMA Journal. 2011; 1: 35-8.

11. Beaskoetxea Gómez P, Bermejo Martínez M, Capillas Pérez R, Cerame Pérez S, García Collado F, Gómez Coiduras JM et al. Situación actual sobre el manejo de heridas agudas y crónicas en España: Estudio ATENEA. Gerokomos [Internet]. 2013 [citado 2018 mayo 08]; 24(1):27-31. Disponible en: http://scielo.isciii.es/scielo.php?script=sci_arttext\& pid=S1134928X2013000100006\&lng=es. http://dx. doi.org/10.4321/S1134-928X2013000100006

12. Usher K, Woods C, Brown J, Power T, Lea J, Hutchinson $\mathrm{M}$ et al. Australian nursing students' knowledge and attitudes towards pressure injury prevention: A cross-sectional study, International journal of nursing studies 2018; 81:14-20. 